\title{
Approximation of a wanted flow via topological sensitivity analysis
}

\author{
M. Abdelwahed \\ Department of Mathematics, College of Science, King Saud University, Riyadh 11451 \\ Kingdom of Saudi Arabia
}

\begin{abstract}
We propose an optimization algorithm for the geometric control of fluid flow. the used approach is based on the topological sensitivity analysis method. It consists in studying the variation of a cost function with respect to the insertion of a small obstacle in the domain. Some theoretical and numerical results are presented in $2 \mathrm{D}$ and $3 \mathrm{D}$.
\end{abstract}

Keywords-Sensitivity analysis, topological gradient, shape optimization, Stokes equations.

\section{INTRODUCTION}

$\mathbf{T}$ HE optimal control of fluid flows has long been receiving considerable attention by engineers and mathematicians due to its importance in many applications involving fluid related technology [11], [16]. There is a wealth of literature on optimal control of flows through suction and injection of fluid along domain boundaries, see e.g. [7], [12]. In the context of design, one of the first studies is found in [18]. It is devoted to determine a minimum drag profile submerged in a homogeneous, steady, viscous fluid by using optimal control theories for distributed parameter systems. Next, many shape optimization methods are introduced to determine the design of minimum drag bodies [8], [15], [19], diffusers [5], and airfoils [6], [17]. The majority of works dealing with optimal design of flow domains fall into the category of shape optimization and are limited to determine the optimal shape of an existing boundary.

It is only recently that topological optimization has been developed and used in fluid design problems. It can be used to design features within the domain allowing new boundaries to be introduced into the design. In this context, one of the first approaches is proposed by Borvall and Petersson in [3]. They implemented the relaxed material distribution approach to minimize the power dissipated in Stokes flow. To approximate the no-slip condition along the solid-fluid interface they used a generalized Stokes problem to model fluid flow throughout the domain. Later, this approach has been generalized by Guest and Prévast in [9]. They treated the material phase as a porous medium where fluid flow is governed by Darcy's law. For impermeable solid material, the no-slip condition is simulated by using a small value for the material permeability to obtain negligible fluid velocities at the nodes of solid elements. The flow regularization is expressed as a system of equations; Stokes flow governs in void elements and Darcy flow governs in solid elements.

In this paper, we propose a new, fast and accurate optimization algorithm based on topological sensitivity analysis [1], [2], [10], [13], [14], [20]. It consists in studying the variation of a cost function with respect to a small topological perturbation of the fluid flow domain.

To present the basic idea, let us consider a domain $\Omega \subset \mathbb{R}^{d}, d=2,3$ and a cost function $j(\Omega)=J\left(\Omega, u_{\Omega}\right)$, where $u_{\Omega}$ is the velocity field solution to Stokes problem defined in $\Omega$. For $\varepsilon>0$, let $\Omega_{\varepsilon}=\Omega \backslash \overline{\left(x_{0}+\varepsilon \omega\right)}$ be the fluid domain obtained by inserting a small obstacle $x_{0}+\varepsilon \omega$ in $\Omega$, where $x_{0} \in \Omega$ and $\omega \subset \mathbb{R}^{d}$ is a fixed bounded domain containing the origin, whose boundary $\partial \omega$ is connected and piecewise of class $\mathcal{C}^{1}$. The topological sensitivity analysis method leads to an asymptotic expansion of the function $j$ in the following form:

$$
j\left(\Omega_{\varepsilon}\right)=j(\Omega)+f(\varepsilon) g\left(x_{0}\right)+o(f(\varepsilon)),
$$

where $f(\varepsilon)$ is a scalar positive function going to zero with $\varepsilon$. This expression is called the topological asymptotic expansion and $g$ is called the topological gradient. The function $g$ is very easy to compute. In order to minimize the cost function, the best location to insert a small obstacle in $\Omega$ is where $g$ is negative. In fact if $g\left(x_{0}\right)<0$, we have $j\left(\Omega_{\varepsilon}\right)<j(\Omega)$ for small $\varepsilon$. Starting with this observation, a topological optimization algorithm can then be constructed. The optimal design is obtained using an iterative process building a sequence of geometries $\left(\Omega_{k}\right)_{k}$ with $\Omega_{0}=\Omega$. At the $k^{t h}$ iteration the topological gradient $g_{k}$ is computed in $\Omega_{k}$ and the new geometry $\Omega_{k+1}$ is obtained by inserting an obstable $\omega_{k}$ in the domain 
$\Omega_{k} ; \Omega_{k+1}=\Omega_{k} \backslash \overline{\omega_{k}}$. The obstacle $\omega_{k}$ is defined by a level set curve of $g_{k}$

$\omega_{k}=\left\{x \in \Omega_{k}\right.$, such that $\left.g_{k}(x) \leq c_{k}<0\right\}$,

where $c_{k}$ is chosen in such a way that the cost function $j$ decreases as most as possible. This algorithm can be seen as a descent method where the descent direction is determined by the topological sensitivity $g_{k}$ and the step length is given by the volume variation meas $\left(\Omega_{k} \backslash \Omega_{k+1}\right)$.

The paper is organized as follows. In section 2, we give a statement of the optimization problem. Section 3 is devoted to a topological sensitivity analysis for the Stokes equations. The obtained results are valid for a large class of cost functions. Similar analysis is developed by Guillaume and SidIdris in [10]. Their approach is based on an adaptation of the adjoint method and a domain truncation technique that provides an equivalent formulation of the PDE in a fixed functional space. In this work, we derive a simplified topological sensitivity analysis for the Stokes equations without using the truncation technique. In section 4 , we present some numerical experiments showing the efficiency of our approach.

\section{TOPOLOGICAL OPTIMIZATION PROBLEM}

Consider a viscous incompressible fluid flow in a bounded domain $\Omega \subset \mathbb{R}^{d}, d=2,3$. We assume that the fluid flow is governed by the Stokes equations.

We denote by $\Omega \backslash \overline{\omega_{\varepsilon}}$ the perturbed domain, obtained by inserting a small obstacle $\omega_{\varepsilon}=x_{0}+\varepsilon \omega$ in the initial domain flow $\Omega$. In $\Omega \backslash \bar{\omega}_{\varepsilon}$, the velocity $u_{\varepsilon}$ and the pressure $p_{\varepsilon}$ are solution to

$$
\left\{\begin{array}{rll}
-\nu \Delta u_{\varepsilon}+\nabla p_{\varepsilon} & =F & \text { in } \Omega \backslash \overline{\omega_{\varepsilon}} \\
\operatorname{div} u_{\varepsilon}=0 & \text { in } \Omega \backslash \overline{\omega_{\varepsilon}} \\
u_{\varepsilon}=0 & \text { on } \Gamma \\
u_{\varepsilon}=0 & \text { on } \partial \omega_{\varepsilon} .
\end{array}\right.
$$

where $\nu$ is the (constant) fluid kinematic viscosity, and $F$ is a given body force per unit of mass. Note that for $\varepsilon=0,\left(u_{0}, p_{0}\right)$ is solution to

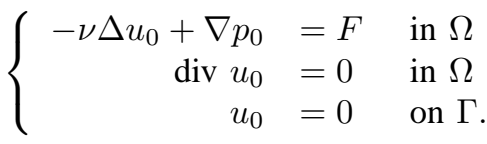

Consider now a design function $j$ of the form

$$
j\left(\Omega \backslash \overline{\omega_{\varepsilon}}\right)=J_{\varepsilon}\left(u_{\varepsilon}\right),
$$

where $J_{\varepsilon}$ is defined on $H^{1}\left(\Omega \backslash \overline{\omega_{\varepsilon}}\right)^{d}$ for $\varepsilon \geq 0$ Our aim is to determine the optimal location of the obstacle $\omega_{\varepsilon}$ in the domain $\Omega$ in order to minimize the cost function $J_{\varepsilon}\left(u_{\varepsilon}\right)$. Then, the optimization problem we consider is given as follows:

$$
\begin{aligned}
& \min _{\omega_{\varepsilon} \subset \Omega} J_{\varepsilon}\left(u_{\varepsilon}\right) \text { such that, for some } p_{\varepsilon}, \\
& \left(u_{\varepsilon}, p_{\varepsilon}\right) \text { is a solution of (1) in } \Omega \backslash \overline{\omega_{\varepsilon}} .
\end{aligned}
$$

To this end, we will derive a topological asymptotic expansion of the function $j$ with respect to $\varepsilon$.

\section{TOPOLOGICAL SENSITIVITY ANALYSIS}

In our topological sensitivity analysis, we have to distinguish the cases $d=2$ and $d=3$. This is due to the fact that the fundamental solutions $(E, \Pi)$ to the Stokes equations in $\mathbb{R}^{2}$ and $\mathbb{R}^{3}$ have essentially different asymptotic behaviour at infinity. We have if $d=3$

$$
\begin{gathered}
E(y)=\frac{1}{8 \pi \nu r}\left(I+e_{r} e_{r}^{T}\right), \\
\Pi(y)=\frac{y}{4 \pi r^{3}}
\end{gathered}
$$

and if $d=2$

$$
\begin{gathered}
E(y)=\frac{1}{4 \pi \nu}\left(-\log (r) I+e_{r} e_{r}^{T}\right), \\
\Pi(y)=\frac{y}{2 \pi r^{2}},
\end{gathered}
$$

with $r=\|y\|, e_{r}=y / r$ and $e_{r}^{T}$ is the transposed vector of $e_{r}$.

Next we assume that $J_{\varepsilon}$ satisfies the following assumption.

Hypothesis 3.1: i) $J_{0}$ is differentiable with respect to $u$, its derivative being denoted by $D J_{0}(u)$. ii) There exists a real number $\delta J$ such that $\forall \varepsilon \geq 0$

$$
\begin{aligned}
J_{\varepsilon}\left(u_{\varepsilon}\right)-J_{0}\left(u_{0}\right)= & D J_{0}\left(u_{0}\right)\left(\widehat{u}_{\varepsilon}-u_{0}\right) \\
& +f(\varepsilon) \delta J+o(\varepsilon),
\end{aligned}
$$

where $f$ is a scalar function and $\widehat{u}_{\varepsilon}$ is an extension of $u_{\varepsilon}$ in $\Omega$ respectively defined by:

$$
\begin{gathered}
f(\varepsilon)= \begin{cases}\varepsilon & \text { if } d=3, \\
-1 / \log (\varepsilon) & \text { if } d=2,\end{cases} \\
\widehat{u}_{\varepsilon}= \begin{cases}u_{\varepsilon} & \text { in } \Omega \backslash \overline{\omega_{\varepsilon}}, \\
0 & \text { in } \omega_{\varepsilon} .\end{cases}
\end{gathered}
$$

A- The three dimensional case: Let $(U, P)$ denotes a solution to

$$
\left\{\begin{aligned}
-\nu \Delta U+\nabla P & =0 & & \text { in } \mathbb{R}^{3} \backslash \bar{\omega} \\
\operatorname{div} U & =0 & & \text { in } \mathbb{R}^{3} \backslash \bar{\omega} \\
U & \longrightarrow 0 & & \text { at } \infty \\
U & =-u_{0}\left(x_{0}\right) & & \text { on } \partial \omega .
\end{aligned}\right.
$$

We start the derivation of the topological asymptotic expansion with the following estimate of the 
$H^{1}\left(\Omega \backslash \overline{\omega_{\varepsilon}}\right)$ norm of $u_{\varepsilon}(x)-u_{0}(x)-U(x / \varepsilon)$. This estimate plays a crucial role in the derivation of our topological asymptotic expansion. It describes the velocity perturbation caused by the presence of the small obstacle $\omega_{\varepsilon}$.

Proposition 3.1: There exists $c>0$, independent of $\varepsilon$, such that for all $\varepsilon>0$ we have

$$
\left\|u_{\varepsilon}(x)-u_{0}(x)-U(x / \varepsilon)\right\|_{1, \Omega \backslash \overline{\omega_{\varepsilon}}} \leq c \varepsilon .
$$

The following corollary follows from Proposition 3.1. It gives the behaviour of the velocity $u_{\varepsilon}$ when inserting an obstacle. The principal term of this perturbation is given by the function $U$, solution to $(6)$.

Corollary 3.1: We have

$u_{\varepsilon}(x)=u_{0}(x)+U(x / \varepsilon)+O(\varepsilon), \quad x \in \Omega \backslash \overline{\omega_{\varepsilon}}$.

We are now ready to derive the topological asymptotic expansion of the cost function $j$. It consists in computing the variation $j\left(\Omega \backslash \overline{\omega_{\varepsilon}}\right)-j(\Omega)$ when inserting a small obstacle inside the domain. The leading term of this variation involves the solution to a boundary integral equation (see Theorem 3.1).

Theorem 3.1: [13] If the assumption 3.1 holds, the function $j$ has the following asymptotic expansion

$$
\begin{aligned}
j\left(\Omega \backslash \bar{\omega}_{\varepsilon}\right)=j(\Omega)+\varepsilon[( & \left.-\int_{\partial \omega} \eta(y) \mathrm{ds}(\mathrm{y})\right) \cdot v_{0}\left(x_{0}\right) \\
& +\delta J]+o(\varepsilon),
\end{aligned}
$$

where $v_{0}$ is the solution to the adjoint problem

$$
\left\{\begin{aligned}
-\nu \Delta v_{0}+\nabla q_{0} & =-D J\left(u_{0}\right) & & \text { in } \Omega \\
\operatorname{div} v_{0} & =0 & & \text { in } \Omega \\
v_{0} & =0 & & \text { on } \Gamma .
\end{aligned}\right.
$$

The function $\eta \in H^{-1 / 2}(\partial \omega)^{3}$ is the solution to the following boundary integral equation

$\int_{\partial \omega} E(y-x) \eta(x) \mathrm{ds}(x)=-u_{0}\left(x_{0}\right), \quad \forall y \in \partial \omega$.

In the particular case where $\omega=B(0,1)$, the density $\eta$ is given explicitly $\eta(y)=$ $-\frac{3 \nu}{2} u_{0}\left(x_{0}\right), \quad \forall y \in \partial \omega$.

Corollary 3.2: If $\omega=B(0,1)$, under the assumption 3.1 we have

$$
\begin{gathered}
j\left(\Omega \backslash \overline{\omega_{\varepsilon}}\right)=j(\Omega)+\varepsilon\left[6 \pi \nu u_{0}\left(x_{0}\right) \cdot v_{0}\left(x_{0}\right)+\delta J\right] \\
+o(\varepsilon) .
\end{gathered}
$$

B- The two dimensional case: In the two dimensional case we have the following asymptotic expansion.
Theorem 3.2: If the assumption 3.1 holds, $j$ admits the following asymptotic expansion

$$
\begin{array}{r}
j\left(\Omega \backslash \overline{\omega_{\varepsilon}}\right)=j(\Omega)+\quad \frac{-1}{\log (\varepsilon)}\left[4 \pi \nu u_{0}\left(x_{0}\right) \cdot v_{0}\left(x_{0}\right)\right. \\
+\delta J]+o\left(\frac{-1}{\log (\varepsilon)}\right) .
\end{array}
$$

\section{NUMERICAL EXAMPLES}

We consider a tank $\Omega$ filled with a viscous and incompressible fluid. The aim is to determine the optimal shape of the fluid flow domain minimizing a given objective function.

Our implementation is based on the following optimization algorithm. We apply an iterative process to build a sequence of geometries $\left(\Omega_{k}\right)_{k \geq 0}$ with $\Omega_{0}=\Omega$. At the $k^{t h}$ iteration the topological gradient $g_{k}$ is computed in $\Omega_{k}$ and the new geometry $\Omega_{k+1}$ is obtained by inserting an obstacle $\omega_{k}$ in the domain $\Omega_{k} ; \Omega_{k+1}=\Omega_{k} \backslash \overline{\omega_{k}}$. The obstacle $\omega_{k}$ is defined by a level set curve of $g_{k}$

$\omega_{k}=\left\{x \in \Omega_{k}\right.$, such that $\left.g_{k}(x) \leq c_{k}<0\right\}$,

where $c_{k}$ is chosen in such a way that the cost function $j$ decreases as much as possible.

\section{The algorithm :}

- Initialization: choose $\Omega_{0}=\Omega$, and set $k=0$.

- Repeat until $g_{k} \geq 0$ in $\Omega_{k}$ :

- solve the Stokes equations in $\Omega_{k}$,

- solve the associated adjoint problem in $\Omega_{k}$,

- compute the topological sensitivity $g_{k}(x)$ $\forall x \in \Omega_{k}$,

- determine the obstacle $\omega_{k}$,

- set $\Omega_{k+1}=\Omega_{k} \backslash \overline{\omega_{k}}$,

- $k \longleftarrow k+1$.

This algorithm can be seen as a descent method where the descent direction is determined by the topological sensitivity $g_{k}$ and the step length is given by the volume variation meas $\left(\Omega_{k} \backslash \Omega_{k+1}\right)$. The natural optimality condition $g_{k}(x) \geq 0, \forall x \in$ $\mathcal{O}_{k}$ is used as stopping criteria [4].

Approximation of a wanted flow. The aim is to determine the optimal shape $\mathcal{O}^{*} \subset \Omega$ of the fluid flow domain such that the velocity $u_{\mathcal{O}^{*}}$, solution to the Stokes equations in $\mathcal{O}^{*}$, approximate a wanted flow $w_{d}$ defined in a fixed domain $\Omega_{m} \subset \Omega$. The optimal shape $\mathcal{O}^{*}$ can be characterized as the solution to the following topological optimization problem

$$
\min _{\mathcal{O} \subset \Omega} \int_{\Omega_{m}}\left|u_{\mathcal{O}}-w_{d}\right|^{2} \mathrm{dx}
$$

where $u_{\mathcal{O}}$ is the solution to the Stokes equations in $\mathcal{O} \subset \Omega$. This test is treated in two and three dimensional cases. In $2 \mathrm{D}$, the tank $\Omega=[0,1.5] \times$ 
$[0,1]$, the domain $\Omega_{m}=[0,1.5] \times[0.8,1]$ and the velocity field $w_{d}$ is defined by: $w_{d}=(1,0)$ in $\Omega_{m}$ and $w_{d}=(0,0)$ elsewhere. The numerical results are described in Figure 1. A 3D extension of this case is presented in Figure 2.

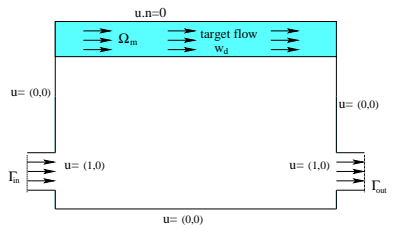

(a) The initial geometry $\Omega$

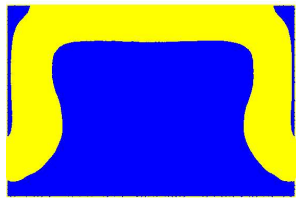

(c) The optimal domain is obtained in only 3 iterations

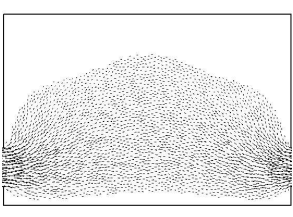

(b) The velocity field in the initial domain

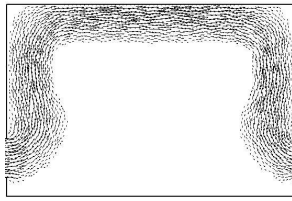

(d) The velocity field in the obtained domain

Fig. 1. Approximation of a wanted flow: 2D case

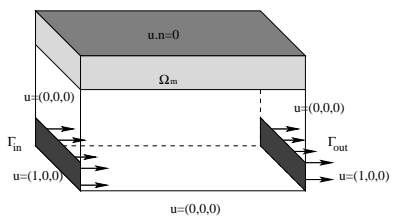

(a) The initial geometry

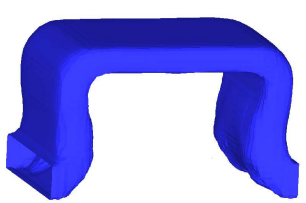

(c) The optimal domain is obtained in only 4 iterations

Fig. 2. Approximation of a wanted flow: 3D case

\section{REFERENCES}

[1] G. Allaire, F. Jouve, A-M. Toader, Structural optimization using sensitivity analysis and a level-set method, J. Comput. Phys., 194 (1), 2004, 363-393.

[2] M. Bendsoe , Optimal topology design of continuum structure: an introduction, Technical report, Departement of mathematics, Technical University of Denmark, DK2800 Lyngby, Denmark, 1996.
[3] T. Borrvall, J. Petersson, Topological optimization of fluids in Stokes flow, Inter. J. Numer. Methods Fluids, 41 (1), 2003, 77-107.

[4] G. Buttazzo, G. Dal Maso , Shape optimization for Dirichlet problems: Relaxed formulation and optimality conditions, Appl. Math. Optim., 23, 1991, 17-49.

[5] H. Cabuk, V. Modi , Optimum plane diffusers in laminar flow, J. of Fluid Mechanics, 237, 1992, 373-393.

[6] E.M. Cliff, M. Heinkenschloss, A. Shenoy, Airfoil design by an all-at-once method, Inter. J. Compu. Fluid Mechanics, 11, 1998, 3-25.

[7] O. Ghattas, J-H. Bark , Optimal control of two and three dimensional incompressible Navier-Stokes flows, Journal of Computational Physics, 136, 1997, 231-244.

[8] R. Glowinski, O. Pironneau , On the numerical computation of the minimum drag profile in laminar flow, J. of Fluid Mechanics, 72, 1975, 385-389.

[9] J. K. Guest, J. H. Prévost , Topology optimization of creeping fluid flows using a Darcy-Stokes finite element, Inter. J. Numer. Methods in Engineering, 66, 2006, 461484.

[10] Ph. Guillaume, K.Sid Idris , Topological sensitivity and shape optimization for the Stokes equations, SIAM J. Control Optim., 43(1), 2004, 1-31.

[11] M.D. Gunzburger , Perspectives in flow control and optimization, Advances in Design and Control, SIAM, Philadelphia, PA, 2003.

[12] M. Gunzburger, H. Kim, S. Manservisi, On a shape control problem for the stationary Navier-Stokes equations, M2AN Math. Model. Numer. Anal. , 34 (6), 2000, 1233 1258 .

[13] M. Hassine, S. Jan, M. Masmoudi , From differential calculus to $0-1$ topological optimization, SIAM J. Cont. Optim., 45 (6), 2007, 1965-1987.

[14] M. Hassine, M. Masmoudi , The topological sensitivity analysis for the Quasi-Stokes problem, ESAIM, COCV J., 10, 2004, 478-504.

[15] D.W. Kim, M.U. Kim , Minimum drag shape in two twodimensional viscous flow, Inter. J. Numer. Methods in Fluids, 21, 1995, 93-111.

[16] B. Mohammadi, O. Pironneau, Applied shape optimization for fluids, Numerical Mathematics and Scientific Computation, Oxford University Press, New York, 2001.

[17] O. Pironneau, On optimum profiles in Stokes flow, J. of Fluid Mechanics, 59, 1973, 117-128.

[18] O. Pironneau, On optimal design in fluid mechanics, J. Fluid Mech., 64, 1974, 97-110.

[19] O. Pironneau, Optimal Shape Design for Elliptic Systems, Springer, Berlin, 1984.

[20] J. Sokolowski, A. Zochowski, On the topological derivative in shape optimization, SIAM J. Control Optim., 37 (4), 1999, 1251-1272.

\section{Creative Commons Attribution License 4.0 (Attribution 4.0 International, CC BY 4.0)}

This article is published under the terms of the Creative Commons Attribution License 4.0 https://creativecommons.org/licenses/by/4.0/deed.en US 